\title{
Actividad antifúngica de extractos orgánicos del árbol Fagara monophylla (Rutaceae) en Venezuela
}

\author{
Yrma Gómez, Katiuska Gil, Elba González \& Luz Marina Farías \\ Dpto. de Ciencias, Laboratorio de Investigaciones Biológicas, Universidad de Oriente, Núcleo de Anzoátegui, \\ Venezuela; irmagomez52@hotmail.com. Fax: 058-0281-2819239
}

Recibido 27-II-2006. Corregido 12-III-2007. Aceptado 27-IV-2007.

\begin{abstract}
Anti-fungi activity of organic extracts from the tree Fagara monophylla (Rutaceae) in Venezuela. The tree Fagara monophylla ranges throughout Tropical America. The genus Fagara has a diversity of alkaloid compounds with antibiotic properties; nevertheless, there are few reports antifungal activity of its organic compounds. Organic extracts from Venezuelan $F$. monophylla were tested for antimicrobial activity against Aspergillus terreus, A. flavus, Penicillium digitatum, P. funiculosum, P. citrinum, Paecilomyces and Candida albicans. Minimal Inhibitory Concentration (MIC) was determined. The susceptibility trials of organic fractions (Hex., $\mathrm{CH}_{2} \mathrm{Cl}_{2}$ and $\mathrm{MeOH}$ ) showed that the highest inhibition was presented by $\mathrm{MeOH}$ against A. flavus (55 $\mathrm{mm})$, P. digitatum $(60 \mathrm{~mm})$, P. funiculosum $(56 \mathrm{~mm})$ and C. albicans $(26 \mathrm{~mm})$. The activities of MeOH/EtOAc fractions 1 and 2 suggest a combined effect against $A$. flavus, $P$. digitatum and $P$. funiculosum. The MIC of 1 $\mathrm{MeOH} /$ EtOAc subfraction activity was lower against $C$. albicans $(32 \mu \mathrm{g} / \mathrm{ml})$ and moderate $(128 \mu \mathrm{g} / \mathrm{ml})$ against P. digitatum. This organic extract has a great antifungal potential. The phytochemical proves and TLC testing on the organic extract, and the $\mathrm{MeOH} / \mathrm{EtOAc}$ subfraction, respectively, indicated the presence of alkaloid compounds. Rev. Biol. Trop. 55 (3-4): 767-775. Epub 2007 December, 28.
\end{abstract}

Key words: antifungal activity, Fagara monophylla, fungi, MIC, organic extract.

Los géneros Fagara y Zantoxylum (Rutaceae), se encuentran tan estrechamente emparentados, que ha resultado problemática la clasificación taxonómica de ambos géneros (Stermitz y Sharifi 1980, De García et al. 1989). Estos géneros se habían mantenido históricamente separados, por las estructuras del perianto de ambos taxones (Stermitz et al. 1980). No obstante, Zantoxylum monophyllum ha sido clasificada como Fagara monophylla y posteriormente reclasificada como Z. monophyllum P. (Wilson) por lo que se consideran sinónimos (Steyermark y Huber 1978, Index Kewensis Plantarum Fanerogamum 1896, Huber et al. 1998).

Las especies de Fagara, son conocidas en la medicina tradicional por sus propiedades como sudorífero, diurético, salivadoras, estimulantes y vomitivas (Reisch et al. 1994). En la medicina tradicional venezolana, es conocido el uso de F. monophylla en el tratamiento del romadizo o inflamación de la mucosa nasal, la ictericia y la oftalmia (Etnobot DB 1999)

F. monophylla conocida en la etnobotánica como paneque, bosuga o bosúa es un árbol que crece hasta $10 \mathrm{~m}$ de altura, caracterizado por ser aromático y poseer ramas espinosas, hojas alternas y flores en panículos terminales. Está distribuida en América Tropical Continental y en algunas de las Antillas (Schenee 1984). Ésta junto con F. pterota, F. chiloperone y F. caribea, constituyen el género Fagara existente en Venezuela (Huber et al. 1998).

Los estudios fitoquímicos del género Fagara, indican que en su composición química existe una diversidad de alcaloides (NAPRALERT DB 2001). Específicamente, F. monophylla (Z. monophyllum) posee un 
alto contenido de alcaloides cuaternarios, en especial berberinas y piranoquinolinas zantofilinas (Stermitz y Sharif 1977, Stermitz et al. 1979, De García et al. 1989). A muchos de los alcaloides presentes en este género se les han atribuido propiedades antibióticas (Couillerot 1996). No obstante, hasta ahora, sólo se le conocen propiedades antifúngicas a las especies $F$. chalybea, F. holstii, F. holtziana, $F$. lemairei y $F$. zantoxyloides (NAPRALERT DB 2001), y poco se conoce acerca de estas propiedades en F. monophylla, por lo que el objetivo de esta investigación fue el de evaluar in vitro la actividad antifúngica de los extractos orgánicos presentes en la corteza de esta planta, con el fin de contribuir al conocimiento de la etnomédica de este género

\section{MATERIALES Y MÉTODOS}

Recolección de la muestra y obtención de los extractos orgánicos: F. monophylla fue recolectada en la localidad de Sabana de Uchire $\left(10^{\circ} 02^{\prime} 00^{\prime \prime} \mathrm{N}, 65^{\circ} 31^{\prime} 00^{\prime \prime} \mathrm{W}\right)$, Estado Anzoátegui, Venezuela, la muestra se encuentra depositada en el Herbario IRBR de la Universidad de Oriente, Venezuela; registrada con el número de colección 1794 (7 de Junio de 1983).

El material vegetal (2 kg) se secó a la sombra durante siete días a temperatura ambiente $\left(26 \pm 3{ }^{\circ} \mathrm{C}\right)$. Las muestras se sometieron a un proceso de maceración con metanol al $99.9 \%$ durante un período de $24 \mathrm{~h}$. La extracción fue repetida hasta agotamiento. Este extracto crudo fue filtrado, concentrado a presión reducida en un rotoevaporador $\left(65 \mathrm{~cm} \mathrm{Hg} / 39^{\circ} \mathrm{C}\right)$ y luego sometido a un proceso de partición con la mezcla de disolventes agua/butanol (1:1). Este proceso permitió la separación de las fracciones orgánica y acuosa. La fracción acuosa fue descartada y la fracción orgánica fue seleccionada para su posterior fraccionamiento mediante la técnica de cromatografía líquida al vacío (VLC) (Coll y Bowden 1986) empleando disolventes de diferentes polaridades: hexano (Hex), diclorometano $\left(\mathrm{CH}_{2} \mathrm{Cl}_{2}\right)$ y metanol $(\mathrm{MeOH})$.
Las fracciones Hex., $\mathrm{CH}_{2} \mathrm{Cl}_{2}$ y $\mathrm{MeOH}$ obtenidas, se concentraron a presión reducida (45-59 y $55 \mathrm{~cm}$ de $\mathrm{Hg} / 39^{\circ} \mathrm{C}$, respectivamente).

A cada una de las fracciones (fr.) orgánicas le fue probada su actividad antifúngica (Bauer et al. 1966). Las fracciones bioactivas se sometieron a un posterior fraccionamiento mediante VLC con gel de sílice, malla 70-230 (Merck), utilizando sistemas de disolventes con gradientes de polaridad (Pelletier 1986). Los eluatos obtenidos de cada fraccionamiento y que mostraron características similares por cromatografía de capa fina (TLC), fueron mezclados (Touchstone 1992). La TLC se realizó sobre placas de gel de silice 60F-245 de $5 \mathrm{~mm}$ de espesor. Las placas se revelaron con luz UV (245 $\mathrm{mn}$ ) y el empleo de una solución reveladora de $\mathrm{H}_{2} \mathrm{SO}_{4}$ al $10 \%$ en metanol, seguido de calentamiento.

La mezcla de los eluatos originó las fracciones: $\mathrm{fr}_{1}$ y fr${ }_{2} \mathrm{Hex} / \mathrm{EtOAc}, \mathrm{fr}_{1}$ y $\mathrm{fr}_{2} \mathrm{CH}_{2} \mathrm{Cl}_{2}$ $/ \mathrm{MeOH}$ y $\mathrm{fr}_{1}$ y $\mathrm{fr}_{2} \mathrm{MeOH} /$ EtOAc. La $\mathrm{fr}_{1}$ $\mathrm{MeOH} / \mathrm{EtOAc}$, fue seleccionada para llevar a cabo un nuevo fraccionamiento (EtOAc $100 \%$ a $\mathrm{MeOH} 100 \%$ ), originando las subfracciones (sf.) $\mathrm{sf}_{1}$ y $\mathrm{sf}_{2} \mathrm{MeOH} / \mathrm{EtOAc}$ (Fig. 2). Ambas subfracciones fueron sometidas a las pruebas de susceptibilidad y determinada la CMI a la $\mathrm{sf}_{1} \mathrm{MeOH} /$ EtOAc.

Un diagrama de flujo del proceso de extracción de los extractos orgánicos de $F$. monophylla se presenta en las Fig. 1 y 2.

Determinación de la actividad antifúngica: se emplearon cultivos puros de diferentes especies de hongos de los géneros Aspergillus: A. terreus (ATCC 10020), A. flavus (ATCC 10124), Penicillium: $P$. digitatum (ATCC 10030), P. citrinum (ATCC 1109), P. funiculosum (NRRL 1033), Paecilomyces sp. y Cándida albicans provenientes de los Laboratorios de Investigaciones Biológicas, y de Microbiología y Parasitología de la Universidad de Oriente.

Esporas de colonias jóvenes de siete días de crecimiento, se suspendieron homogéneamente en una solución de peptona al $0.1 \%$. A partir de esta suspensión se realizó un sistema de dilución hasta asegurar una concentración de $4 \times 10^{4}$ unidades formadoras de colonias 


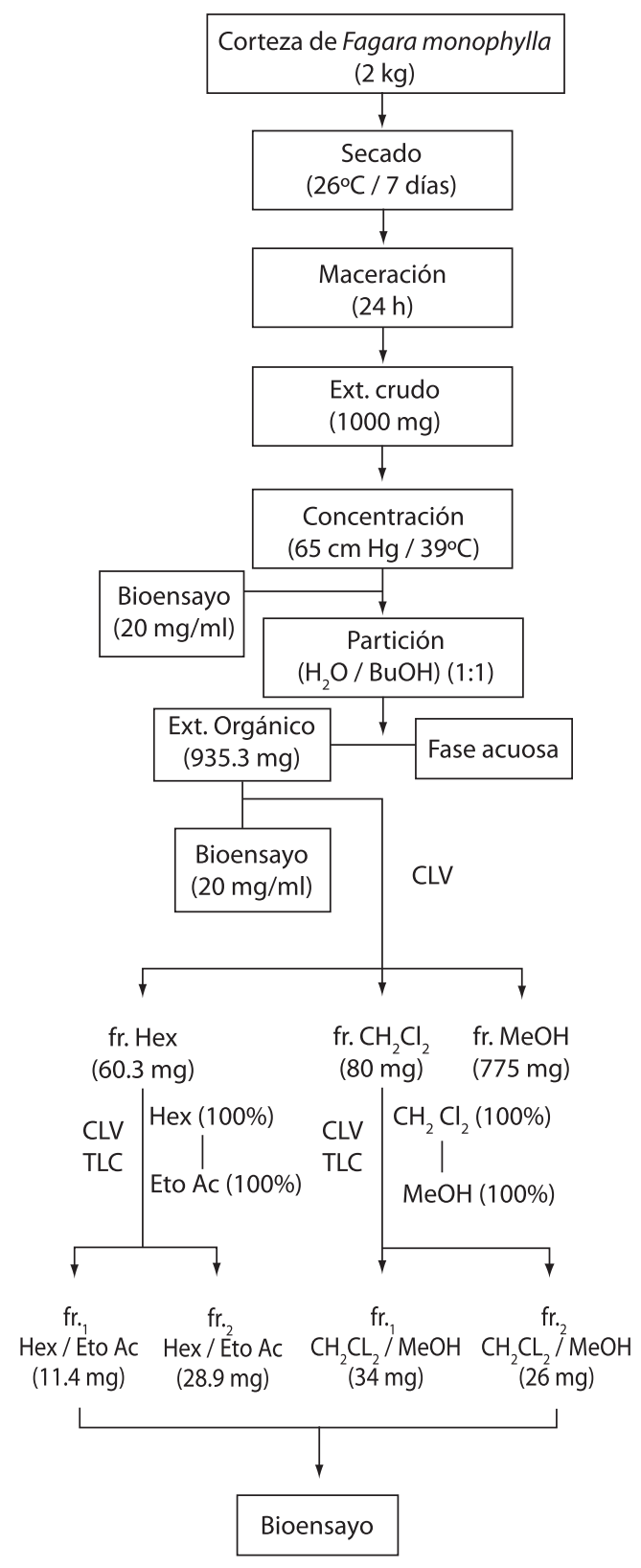

Fig. 1. Proceso de obtención de las fracciones Hex y $\mathrm{CH}_{2} \mathrm{Cl}_{2}$ de Fagara monophylla.

Fig. 1. Extraction process for fractions $\mathrm{Hex}$ and $\mathrm{CH}_{2} \mathrm{Cl}_{2}$ of Fagara monophylla.
(UFC/ml) (Kobayashi et al. 1996). $0.1 \mathrm{ml}$ de esta suspensión se inoculó en la superficie del agar Czapex dox para el caso de hongos filamentosos y agar Sabureaud para C. albicans. El crecimiento bacteriano se inhibió mediante la adición de $1 \mathrm{ml} / \mathrm{l}$ de cloranfenicol $(0.5 \mathrm{mg} / \mathrm{ml})$ al medio de cultivo.

Los discos impregnados con $20 \mu \mathrm{l}$ del extracto $(20 \mathrm{mg} / \mathrm{ml})$ se colocaron asépticamente sobre el medio de cultivo previamente inoculado con $0.1 \mathrm{ml}$ de una suspensión esporangial. Los medios inoculados fueron enfriados a $4{ }^{\circ} \mathrm{C}$ durante $12 \mathrm{~h}$ previo a su incubación a $27^{\circ} \mathrm{C}$, a excepción de C. albicans la cual fue incubada a $37{ }^{\circ} \mathrm{C}$ durante $48 \mathrm{~h}$.

La actividad antifúngica se determinó por la presencia de un halo de inhibición alrededor del disco. Los resultados se expresaron como el diámetro $(\mathrm{mm})$ del halo de inhibición (Bauer et al. 1966). Como controles negativos se utilizaron discos impregnados con el disolvente empleado para la obtención del extracto.

Determinación de la concentración mínima inhibitoria: los extractos bioactivos fueron disueltos en DMSO al $10 \%$ en agua y diluidos en forma seriada a concentraciones comprendidas entre 0.125 y $512 \mu \mathrm{g} / \mathrm{ml}$. En cada caso, éstas fueron añadidas al medio de cultivo en una relación 1:9 (Delgado et al. 1994). El inóculo fue preparado a partir de un caldo nutritivo ajustado a una concentración de $10^{-4} \mathrm{UFC} / \mathrm{ml}$. Las placas fueron incubadas a $27^{\circ} \mathrm{C}$ durante 48 $\mathrm{h}$ y el crecimiento microbiano fue inspeccionado visualmente. La CMI fue definida como la más baja concentración del compuesto que previene cualquier crecimiento visible (Jawetz et al. 1992).

Pruebas fitoquímicas: al extracto orgánico se le realizaron las pruebas fitoquímicas para la identificación de las familias de compuestos pertenecientes a las saponinas, alcaloides, flavonoides, polifenoles, triterpenos, esteroles y taninos, según las técnicas descritas por Marcano y Hasegawa (1991). La técnica de 


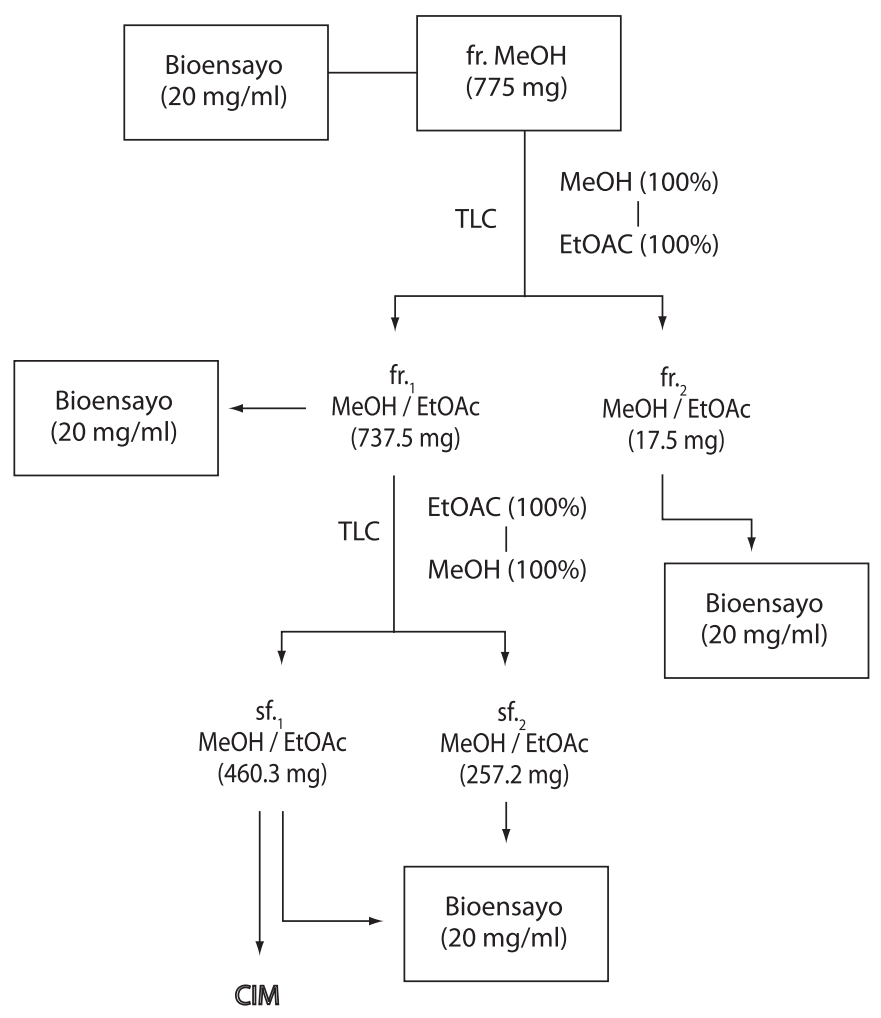

Fig. 2. Proceso de obtención de la fracción MeOH de Fagara monophylla.

Fig. 2. Extraction process for fraction $\mathrm{MeOH}$ of Fagara monophylla. albicans (24 mm), y una actividad fungistática frente a $P$. funiculosum (Cuadro 1).

El fraccionamiento del extracto orgánico por VLC con disolventes de diferentes polaridades produjo las siguientes fracciones (fr.): fr. Hex (60.3 $\mathrm{mg})$; fr. $\mathrm{CH}_{2} \mathrm{Cl}_{2}(80 \mathrm{mg})$ y la fr. $\mathrm{MeOH}$ (775 mg) (Fig. 1). Las pruebas de susceptibilidad realizadas a estas fracciones se muestran en el Cuadro 2. Se observa, que la fr. Hex provocó inhibición frente a todos los hongos ensayados. La fr. $\mathrm{CH}_{2} \mathrm{Cl}_{2}$ sólo provocó la inhibición de P. funiculosum (27 $\mathrm{mm})$ seguido de $P$. digitatum (25 mm) y A. flavus $(22 \mathrm{~mm})$. Mientras que fr. $\mathrm{MeOH}$ presentó la mayor actividad antifúngica frente a $P$. digitatum (60 mm), P. funiculosum (56 $\mathrm{mm})$, A. flavus $(55 \mathrm{~mm})$ y $C$. albicans. $(26 \mathrm{~mm})$.

El fraccionamiento cromatográfico de la fr. Hex. la TLC y el uso del reactivo de Dragendorff, revelaron la presencia de alcaloides en la $\mathrm{sf}_{1}$ $\mathrm{MeOH} / \mathrm{EtOAc}$.

\section{RESULTADOS}

A partir del macerado de $F$. monophylla se obtuvieron $1000 \mathrm{mg}$ del extracto crudo, el cual resultó bioactivo frente a todos los organismos ensayados (Cuadro 1). El extracto una vez particionado con la mezcla de butanol/ $\mathrm{H}_{2} \mathrm{O}(1: 1)$ resultó en la formación de un extracto orgánico (935.3 mg) y una fase acuosa (Fig. 1). $20 \mu 1$ $(20 \mathrm{mg} / \mathrm{ml})$ del extracto orgánico se emplearon para realizar las pruebas de susceptibilidad. Estas pruebas mostraron, que el extracto orgánico sólo presentó actividad antifúngica frente a A. flavus $(30 \mathrm{~mm})$, P. digitatum $(40 \mathrm{~mm}) C$.

\section{CUADRO 1}

Actividad antifúngica del extracto crudo y orgánico de Fagara monophylla contra diferentes especies fúngicas

TABLE 1

Antifungal activity of crude and organic extracts of Fagara monophylla against different fungi species

\begin{tabular}{lcc} 
Microorganismos & \multicolumn{2}{c}{ Diámetro del halo de inhibición (mm) } \\
& Extracto crudo & Extracto orgánico \\
A. flavus & 21 & 30 \\
A. terreus & 40 & - \\
P. citrinum & 20 & - \\
P. digitatum & 40 & 40 \\
P. funiculosum & 20 & $27 / 10^{*}$ \\
Paecilomyces sp. & 30 & - \\
C. albicans & 32 & 24 \\
*Zona de inhibición total/diámetro del halo de inhibición.
\end{tabular}


CUADRO 2

Actividad antifúngica de las fracciones $\mathrm{Hex}, \mathrm{CH}_{2} \mathrm{Cl}_{2}$ y $\mathrm{MeOH}$ de Fagara monophylla contra diferentes especies fúngicas

TABLE 2

Antifungal activity of $\mathrm{Hex}, \mathrm{CH}_{2} \mathrm{Cl}_{2}$ and $\mathrm{MeOH}$ fractions from Fagara monophylla against different fungi species

Microorganismos

Diámetro del halo de inhibición (mm) fr. Hex fr. $\mathrm{CH}_{2} \mathrm{Cl}_{2}$ fr. $\mathrm{MeOH}$

A. flavus

A. terreus

$$
24
$$

22 55

P. citrinum

P. digitatum

P. funiculosum

Paecilomyces sp.

C. albicans

\section{5}

16

$$
26
$$

25

60

31

27

35

10

(60.3 mg) mediante un sistema de disolventes de polaridad creciente (100\% Hex a $100 \%$ EtOAc), originó las fracciones: $\mathrm{fr}_{1} \mathrm{y} \mathrm{fr}_{2} \mathrm{Hex}$ /EtOAc (Fig. 1), las cuales resultaron inactivas (Cuadro 3).

El fraccionamiento de la fr. $\mathrm{CH}_{2} \mathrm{Cl}_{2}(80$ mg) mediante CLV, empleando la mezcla de disolventes: $\mathrm{CH}_{2} \mathrm{Cl}_{2} / \mathrm{MeOH}\left(100 \% \mathrm{CH}_{2} \mathrm{Cl}_{2}\right.$, a $100 \% \mathrm{MeOH}$ ), originó las fracciones $\mathrm{fr}_{\mathrm{I}} \mathrm{y} \mathrm{fr}_{2}$ $\mathrm{CH}_{2} \mathrm{Cl}_{2} / \mathrm{MeOH}$ (34 mg y $26 \mathrm{mg}$ respectivamente) (Fig. 1).

Las pruebas de susceptibilidad de las fracciones $\mathrm{fr}_{1} \mathrm{y} \mathrm{fr} \mathrm{CH}_{2} \mathrm{Cl}_{2} / \mathrm{MeOH}$ (Cuadro 3) mostraron que la $\mathrm{fr}_{1} \mathrm{CH}_{2} \mathrm{Cl}_{2} / \mathrm{MeOH}$ sólo inhibió a Paecilomyces sp. (39 mm); mientras que la $\mathrm{fr}_{2} \mathrm{CH}_{2} \mathrm{Cl}_{2} / \mathrm{MeOH}$, inhibió el mayor número de organismos y mostró su mayor actividad frente a $A$. terreus $(43 \mathrm{~mm})$ (Cuadro 3).

El fraccionamiento de la fr. $\mathrm{MeOH}(775$ mg) mediante CLV empleando un sistema de disolventes con un gradiente de polaridad decreciente $(100 \% \mathrm{MeOH}$ a $100 \%$ EtOAc) produjo la formación de las $\mathrm{fr}_{1} \mathrm{y} \mathrm{fr}_{2} \mathrm{MeOH} /$ EtOAc (737.5 mg y $17.50 \mathrm{mg}$, respectivamente) (Fig. 2).

La $\mathrm{fr}_{1} \mathrm{MeOH} / \mathrm{EtOAc}$, presentó actividad antifúngica frente a $A$. flavus $(50 \mathrm{~mm}), P$.
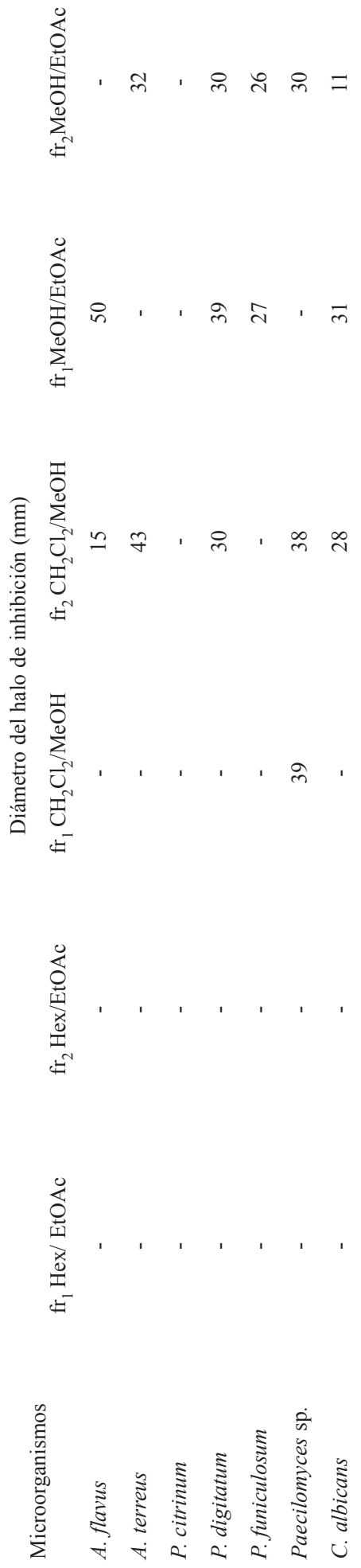
digitatum $(39 \mathrm{~mm})$, C. albicans. $(31 \mathrm{~mm}) \mathrm{y}$ P. funiculosum $(27 \mathrm{~mm})$; mientras que $\mathrm{la} \mathrm{fr}_{2}$ $\mathrm{MeOH} /$ EtOAc, inhibió además de estos organismos, el crecimiento de $A$. terreus $(32 \mathrm{~mm})$ y Paecilomyces sp. (30 mm).

De acuerdo a las cantidades obtenidas para las fracciones $\mathrm{fr}_{1}$ y fr ${ }_{2} \mathrm{CH}_{2} \mathrm{Cl}_{2} / \mathrm{MeOH}$ (34 y 26 $\mathrm{mg}$, respectivamente), y fr ${ }_{1}$ y $\mathrm{fr}_{2} \mathrm{MeOH} / \mathrm{EtOAc}$ (737.5 y $17.5 \mathrm{mg}$, respectivamente), sólo se pudo fraccionar la $\mathrm{fr}_{1} \mathrm{MeOH} /$ EtOAc y para ello, se empleó el sistema de disolventes $\mathrm{MeOH} /$ EtOAc (100\% EtOAc a $100 \% \mathrm{MeOH})$. Como resultado de este proceso, se obtuvieron las subfracciones ( $\mathrm{sf}): \mathrm{sf}_{1}(460.3 \mathrm{mg})$ y $\mathrm{sf}_{2}(257.2$ $\mathrm{mg}$ ) $\mathrm{MeOH} /$ EtOAc (Fig. 2), de las cuales, la $\mathrm{sf}_{1} \mathrm{MeOH} /$ EtOAc mostró los mayores halos de inhibición frente a $P$. digitatum $(45 \mathrm{~mm})$, seguido de $P$. funiculosum $(39 \mathrm{~mm})$; mientras que la $\mathrm{sf}_{2} \mathrm{MeOH} / \mathrm{EtOAc}$, mostró mayor actividad antifúngica frente a $C$. albicans $(46 \mathrm{~mm})$ y $P$. digitatum (40 mm) (Cuadro 4).

Concentración mínima inhibitoria: el Cuadro 5 presenta los valores de la CMI de la subfracción $\mathrm{sf}_{1} \mathrm{MeOH} /$ EtOAc para A. flavus, P. funiculosun, P. digitatum y C. albicans. En el ámbito de las concentraciones empleadas $(0.125-512 \mu \mathrm{g} / \mathrm{ml})$, el $100 \%$ de la inhibición de $C$. albicans ocurrió a una concentración

\section{CUADRO 4}

Actividad antifúngica de las subfracciones $s f_{1}$ y $s f_{2}$ $\mathrm{MeOH} / \mathrm{EtOAc}$ de Fagara monophylla contra differentes especies fúngicas

TABLE 4

Antifungal activity of $s f_{1}$ and $s f_{2} \mathrm{MeOH} / \mathrm{EtOAc}$ fractions of Fagara monophylla against different fungi species

Diámetro del halo de inhibición (mm)

Microorganismos $\begin{array}{cc}\mathrm{sf}_{1} & \mathrm{sf}_{2} \\ \mathrm{MeOH} / \text { EtOAc } & \mathrm{MeOH} / \text { EtOAc }\end{array}$

A. flavus

$$
22
$$

A. terreus

P. citrinum

P. digitatum

P. funiculosum

Paecilomyces sp.

C. albicans
CUADRO 5

Valores de la Concentración Mínima Inhibitoria (CMI) de la subfracción $\mathrm{sf}_{1} \mathrm{MeOH} / \mathrm{EtOAc}$

TABLE 5

Values of Minimal Inhibitory Concentration (MIC) of $\mathrm{sf}_{1} \mathrm{MeOH} / \mathrm{EtOAc}$ subfraction

Microorganismos

Concentration Mínima Inhibitoria $(\mu \mathrm{g} / \mathrm{ml}) \mathrm{sf}_{1} \mathrm{MeOH} / \mathrm{EtOAc}$

$\begin{array}{ll}\text { A. flavus } & >512 \\ \text { P. funiculosum } & >512 \\ \text { P. digitatum } & \geq 128 \\ \text { C. albicans } & \geq 32\end{array}$

CUADRO 6

Pruebas fitoquímicas del extracto orgánico y de la $s f_{l}$ $\mathrm{MeOH} / \mathrm{EtOAc}$ de Fagara monophylla

TABLE 6

Phytochemical test of organic extract and $s f_{1} \mathrm{MeOH} /$ EtOAc of Fagara monophylla

Familias Químicas

Resultados

Saponinas

Alcaloides

Flavonoides

Polifenoles

Triterpenos y Esteroles

Taninos

de $32 \mu \mathrm{g} / \mathrm{ml}$ del extracto, y para $P$. digitatum a $128 \mu \mathrm{g} / \mathrm{ml}$. Las concentraciones de la $\mathrm{sf}_{1}$ $\mathrm{MeOH} /$ EtOAc empleadas para $A$. flavus y $P$. funiculosum, no provocaron la total inhibición de estos organismos.

Las pruebas fitoquímicas realizadas al extracto orgánico y la prueba de Dragendorf realizada a la $\mathrm{sf}_{1} \mathrm{MeOH} / \mathrm{EtOAc}$ revelaron la presencia de alcaloides en ambos extractos (Cuadro 6).

\section{DISCUSIÓN}

Las pruebas de susceptibilidad realizadas al extracto crudo de F. monophylla (Cuadro 1), 
revelaron la presencia de compuestos o familias de compuestos con actividad antifúngica, evidenciando de acuerdo al registro de la base de datos de NAPRALERT DB (2001), que los resultados obtenidos en esta investigación constituyen uno de los primeros reportes de las propiedades antifúngicas presentes en esta planta.

En contraste a la amplia actividad antifúngica presente en el extracto crudo de F. monophylla, las pruebas de susceptilidad realizadas a la fracción orgánica, sólo resultaron positivas contra A. flavus $(30 \mathrm{~mm})$, P. digitatum (40 $\mathrm{mm}$ ), y C. albicans. (24 mm) (Cuadro 1), lo cual sugiere la presencia de un posible efecto sinérgico. Es probable, que la acción conjunta de diferentes sustancias presentes en el extracto crudo, pueda potenciar el efecto antifúngico, en contraste al menor efecto observado cuando estas sustancias actúan separadamente (Hardman et al. 1996, Hay y Fenical 1996, Fauci et al. 1998).

La fracción Hex. inhibió el crecimiento de todos los organismos estudiados (Cuadro 2), lo cual indica, que los compuestos orgánicos de baja polaridad presentes en esta fracción son capaces de inhibir el crecimiento de estos organismos. Mientras que las pruebas de susceptibilidad realizadas a la fr. $\mathrm{CH}_{2} \mathrm{Cl}_{2}$ y fr. $\mathrm{MeOH}$ (Cuadro 2), afectaron a un menor número de éstos; efecto que pudiera estar relacionado con algunos de los mecanismos de acción de las sustancias antifúngicas, como es la posible alteración de la permeabilidad de la membrana y su afinidad por ella (Vanden-Bossche 1997).

El fraccionamiento de la fr. Hex $(60.3 \mathrm{mg})$ condujo a una pérdida de la actividad antifúngica presente en los compuestos de esta fracción (Cuadro 3), lo cual podría explicarse debido a un efecto sinérgico entre los compuestos de las fracciones $\mathrm{fr}_{1}$ y fr ${ }_{2} \mathrm{Hex} /$ EtOAc (11.4 y $28.9 \mathrm{mg}$, respectivamente) (Cuadro 2 ).

La fr ${ }_{2} \mathrm{CH}_{2} \mathrm{Cl}_{2} / \mathrm{MeOH}$ presentó la mayor actividad antifúngica; mientras que la $\mathrm{fr}_{1}$ $\mathrm{CH}_{2} \mathrm{Cl}_{2} / \mathrm{MeOH}$ (34 mg) prácticamente no mostró este tipo de actividad (Cuadro 3). Las diferencias en actividad entre estas fracciones y la actividad mostrada por la fr. $\mathrm{CH}_{2} \mathrm{Cl}_{2}$ de la cual provienen (Cuadro 2), probablemente se deba a que los compuestos existentes en esta última, pudieran ejercer entre sí cierto grado de interferencia o antagonismo (Hardman et al. 1996), que no les permite la máxima expresión de su actividad.

Si se comparan las actividades antifúngicas mostradas por la fr. $\mathrm{MeOH}$ (Cuadro 2) y las $\mathrm{fr}_{1}$ y fr ${ }_{2} \mathrm{MeOH} /$ EtOAc (Cuadro 3), se observa, que el o los compuestos con actividad antifúngica que inhiben el crecimiento de $A$. flavus, están presentes en la $\mathrm{fr}_{1} \mathrm{MeOH} / \mathrm{EtOAc}$. En contraste, A. terreus y Peacilomyces sp., los cuales no fueron inhibidos por la fr. $\mathrm{MeOH}$ (Cuadro 2), resultaron inhibidos por los compuestos presentes en la $\mathrm{fr}_{2} \mathrm{MeOH} /$ EtOAc (Cuadro 3), lo cual sugiere un efecto sinérgico entre los compuestos de ambas fracciones.

A pesar de que la fr. $\mathrm{MeOH}$ provocó un halo de inhibición de $60 \mathrm{~mm}$ frente a $P$. digitatum y de $56 \mathrm{~mm}$ frente a $P$. funiculosum (Cuadro 2), su fraccionamiento condujo a una reducción casi equitativa de su actividad frente a estos organismos (Cuadro 3). De ello es posible inferir, que en la fr. $\mathrm{MeOH}$ existe un efecto combinado de sustancias que potencian su actividad frente a estos organismos (Cuadro 3). Para el caso específico de C. albicans, $\mathrm{la} \mathrm{fr}_{1}$ $\mathrm{MeOH} / \mathrm{EtOAc}$ provocó una mayor inhibición del crecimiento de este organismo $(31 \mathrm{~mm})$.

El Cuadro 4 muestra que la $\mathrm{sf}_{1}$ y $\mathrm{sf}_{2}$ $\mathrm{MeOH} /$ EtOAc presentaron un incremento de la actividad antifúngica para $P$. digitatum (45 y $40 \mathrm{~mm}$ respectivamente) y $C$. albicans $(31 \mathrm{y}$ $46 \mathrm{~mm}$ respectivamente), lo cual sugiere, que el fraccionamiento de la $\mathrm{fr}_{1} \mathrm{MeOH} /$ EtOAc pudiera potenciar la acción antifúngica del extracto resultante para estos organismos.

El halo de inhibición $(46 \mathrm{~mm})$ provocado por la $\mathrm{sf}_{2} \mathrm{MeOH} / \mathrm{EtOAc}$, frente a C. albicans (Cuadro 4), es de particular importancia, si consideramos la resistencia que este organismo presenta actualmente a diferentes antifúngicos: flucytosine, griseofulvina, azoles, (Albertson et al. 1996, Vanden-Bossche 1997) y anfotericina B (Ribaud 1997).

La CMI de la sf ${ }_{1} \mathrm{MeOH} /$ EtOAc, para $C$. albicans, estuvo representada por una concentración de $32 \mu \mathrm{g} / \mathrm{ml}$ (Cuadro 5), la cual la ubica 
dentro de aquellos extractos de plantas que poseen un gran potencial con actividad antifúngica (Rios et al. 1988). Para P. digitatum, la CMI fue de $128 \mu \mathrm{g} / \mathrm{ml}$ (Cuadro 5), concentración considerada como de eficiente grado de inhibición (Cimanga et al. 1996). Las concentraciones mayores de $500 \mathrm{mg} / \mathrm{ml}$ observadas para $A$. flavus y $P$. funiculosum se consideran no activas (Rios et al. 1988).

El Cuadro 6, muestra que la naturaleza química del extracto orgánico de $F$. monophylla, contiene sustancias alcaloides, compuestos que ocurren comunmente entre otras especies del género Fagara y Xanthophyllum (Couillerot et al. 1996, Chen et al. 1996, Deyun et al. 1996). Así mismo, la prueba de Dragendorf reveló la presencia de compuestos alcaloides en la $\mathrm{sf}_{1}$ $\mathrm{MeOH} / \mathrm{EtOAc}$. La presencia de alcaloides en la $\mathrm{sf}_{1} \mathrm{MeOH} /$ EtOAc de F. Monophylla corrobora los informes de Gundiza (1987), quien afirma, que muchos compuestos alcaloides poseen propiedades antibacterianas y antifúngicas.

\section{AGRADECIMIENTOS}

Al Consejo de Investigación de la Universidad de Oriente.

\section{RESUMEN}

Fagara monophylla está distribuida en América Tropical. El género Fagara tiene diversidad de compuestos alcaloides con propiedades antibacterianas; sin embargo existe escasa información acerca de su actividad antifúngica. Evaluamos extractos de $F$. monophylla in vitro con los hongos Aspergillus terreus, A. flavus, Penicillium digitatum, P. citrinum, P. funiculosum, Paecilomyces y Candida albicans determinando la Concentración Mínima Inhibitoria (CMI). Hicimos pruebas de susceptibilidad con las fracciones Hex., $\mathrm{CH}_{2} \mathrm{Cl}_{2}$ y la $\mathrm{MeOH}$. La mayor inhibición la presentó la fracción $\mathrm{MeOH}$ frente a A. flavus $(55 \mathrm{~mm})$, P. digitatum $(60 \mathrm{~mm}), P$. funiculosum $(56 \mathrm{~mm})$ y C. albicans $(26 \mathrm{~mm})$. Las actividades mostradas por las fracciones 1 y 2 de $\mathrm{MeOH} /$ EtOAc sugieren la acción de un efecto combinado frente a A flavus, $P$, digitatum y $P$. funiculosum. La CMI de la subfracción $1 \mathrm{MeOH} /$ EtOAc frente a Candida albicans fue baja $(32 \mu \mathrm{g} / \mathrm{ml})$, y para $P$. digitatum la CMI fue moderada $(128 \mu \mathrm{g} / \mathrm{ml})$. Este extracto orgánico posee gran potencial de actividad antifúngica. Las pruebas fitoquímicas y los ensayos realizados por TLC al extracto orgánico y a la subfracción $1 \mathrm{MeOH} / E t O A c$, respectivamente, mostraron la presencia de alcaloides.

Palabras claves: actividad antifúngica, Fagara monophylla. hongos, MIC, extracto orgánico.

\section{REFERENCIAS}

Albertson, G.D., M. Niimi, R.D. Cannon \& H.F. Jenkinson. 1996. Multiple efflux Mechanisms are involved in Candida albicans fluconazole resistance. Antimicrob. Agente Chemother. 40: 2835-2841.

Bauer, A., W. Kirby, I. Sherris \& M. Turk. 1966. Antibiotic susceptiblility testing by standardized single disk method. Am. J. Clin. Pathol. 45: 493-496.

Chen, H., R.X. Tan, Z.L. Liu \& Y. Zhang. 1996. Antibacterial neoclerodane diterpenoids from Ajuga lupulina. J. Nat. Prod. 59: 663-670.

Cimanga, K., T. De Bruyne, A. Lasure, B. Van Poel, L. Pieters, M. Claeys D. Van den Berghe, K. Kambu, L. Tobna \& A.J. Vlietinck. 1996. In Vitro biological activities of alkaloids from Cryptolepis sanguinolenta. Plant Med. 62: 22- 27.

Coll, J.C. \& B.F. Bowden. 1986. The aplication of vacum liquid chromatography to the separation of terpene mixture. J. Nat. Prod. 49: 934-936.

Couillerot, E., C. Caron, J.C. Audran, J.C. Jordillier \& J.C. Chenieux. 1996. Furoquinoline alkaloids acumulation in Fagara zanthoxyloides cell cultures is highly dependent on the presence of exogenous benzylamino purine. Plant-growth regul. Kluwer Academic Publishers-Dordrecht. 3: 203-206.

De García, L.A, J. Calle \& M.T. Reguero. 1989. Pthytochemical Study of Zanthoxylum monophulum. Fitoterapia 60: 447-450.

Delgado, A., S. Amich, S. Prieto \& M. Salve. 1994. Laboratorio clínico de microbiología. McGraw-Hill, Madrid, España.

Deyun, K., A.I. Gray, T.G. Hartley \& P.G. Waterman. 1996. Alkaloids from an Australian accession of Zanthoxylum. Biochem. Syst. Ecol. 1: 87-88.

Fauci, A.S., E. Brauunwald, K.J. isselbacher, J.D. Wilson, J.B. Martin, D.L. Kasper \& Dan L. Longo. 1998. Harrison: Principios de Medicina Interna. McGraw Hill, México D.F., México. 
Gundiza, M. 1987. Antimicrobial activities of Helinus integrifolius. Fitoterapia 58: 180-183.

Hardman, J.G., L.E. Limbird, P.B. Molinoff, R.W. Ruddon. 1996. Goodman \& Gilman: Las bases farmacológicas de la terapéutuca. McGraw-Hill, México D.F., México.

Hay, M.E. \& W. Fenical. 1996. Chemical ecology and marine biodiversity: insights and products from the sea. Oceanography 9: 10-20.

Huber, O., R. Duno, R.C. Riina, F. Staffer, L. Pappaterra, A. Jimínez, S. Llamozas \& G. Orsini. 1998. Estado Actual del Conocimiento de la Flora en Venezuela. Ministerio del Ambiente de los Recursos Naturales Renovables. Programa de las Naciones Unidas para el Desarrollo, Caracas, Venezuela.

Index Kewensis Plantarum fanerogamun. 1895. Suplementos hasta 1955. Oxford University, Oxford, Inglaterra.

Jawetz, E., J.L. Melnick, C.A. Adelberg, G.F. Broooks, J.S Butel, L.N. Ornston. 1992. Microbiología Médica. Manual Moderno, México D.F., México.

Kobayashi, H., M. Naminoshi, T. Yoshimoto \& T. Yokochit. 1996. A screening method for antimicotic and antifungal sustance using conidia of Pycularia oryzae. Modification and application to tropical marine fungy. J. Antibiotics. 9: 573-879.

Pelletier, S.W. 1986. Separation of diterpenoid, alkaloid mixture using vacuum liquid chromatography. J. Nat. Prod. 49: 892-900.

Reisch, J., N. Brescacin \& A.A. Gurni. 1994. Constituents of Fagara species from Argentina. Fitoterapia 65: 379.
Ribaud, P. 1997. Fungal infections and the cancer patient. Eur J. Cancer. 33: 550-554.

Rios, J.L., M.C. Recio \& A. Villar. 1988. Screening methods for natural products of the literature. J. Phytopharmacol. 23: 127-149.

Schnee, L. 1984. Plantas comunes de Venezuela. Facultad de Agronomía. Universidad Central de Venezuela, Caracas, Venezuela.

Stermitz, F.R. \& I. Sharifi. 1977. Alkaloids of Zanthoxulum monophylum and Z. puntatum. Phytochemistry 16 : 2003-2006.

Stermitz, F.R., M.A. Caolo \& J.A. Swinehart. 1980. Alkaloids and other constituents of Zanthoxyllum Williamsii, Z. Monophyllum and Fagara. Phytochemistry 19: 1464-1472.

Steyermark, J.A \& O. Huber. 1978. Flora del Avila. Flora y Vegetación de las Montañas del Avila de la Silla y del Naiguatá. Publicación especial de la Sociedad Venezolana de las Ciencias Naturales, bajo los auspicios de Vollmer Foundation. Ministerio del Ambiente y de los Recursos Naturales, Caracas, Venezuela.

Touchstone, J. 1992. Practice of thin layer cromatography. Wiley, Nueva York, EEUU.

Vanden,-Bossche, H. 1977. Mechanisms of antifungal resístanse. Rev. Iberoam. Micol. 14: 44-49.

\section{REFERENCIAS INTERNET}

Etnobot, D.B. 1999. Taxon Fagara monophylla. Disponible en http://probenat.usda.gov.8500/egr-brn/webace/ubetnnobotubocrass-Genus $\alpha$ object-Fagara;consultado 7-III-2006. 
